\title{
Improved stability in wide-recess InP HEMTs by means of a fully passivated two-step-recess gate
}

\author{
Tetsuya Suemitsu $^{1 a)}$, Yoshino K. Fukai ${ }^{1}$, Masami Tokumitsu ${ }^{1}$, \\ Fabiana Rampazzo $^{2}$, Gaudenzio Meneghesso ${ }^{2}$, and Enrico Zanoni ${ }^{2}$ \\ ${ }^{1}$ NTT Photonics Laboratories, NTT Corporation \\ 3-1 Morinosato Wakamiya, Atsugi, Kanagawa 243-0198, Japan \\ ${ }^{2}$ Department of Information Engineering, University of Padova \\ 6/B via Gradenigo, Padova 35131, Italy \\ a)sue@aecl.ntt.co.jp
}

\begin{abstract}
We report two-step-recess gate InP HEMTs with a new process option suitable for producing a wide recess. In the new devices the gate recess is completely covered with a passivation film. Though the gate recess is extremely wide, a transconductance of $1 \mathrm{~S} / \mathrm{mm}$ and a cutoff frequency of $208 \mathrm{GHz}$ are achieved with 100-nm gate devices. Moreover, a huge improvement in the drain reliability is achieved by the wide recess which reduces hot-carrier-induced degradation, and by the full passivation which eliminates the instability related to the recess surface.
\end{abstract}

Keywords: HEMT, FET, InP, InGaAs, reliability, cutoff frequency Classification: Electron devices

\section{References}

[1] J. A. del Alamo and A. A. Villanueva, "Thermal, electrical and environmental reliability of InP HEMTs and GaAs PHEMTs," IEDM Tech. Dig., San Francisco, USA, pp. 1019-1022, Dec. 2004.

[2] G. Meneghesso, D. Buttari, E. Perin, C. Canali, and E. Zanoni, "Improvement of dc, low frequency and reliability properties of InAlAs/InGaAs InP-based HEMTs by means of an InP etch stop layer," IEDM Tech. Dig., San Francisco, USA, pp. 227-230, Dec. 1998.

[3] T. Suemitsu, Y. K. Fukai, H. Sugiyama, K. Watanabe, and H. Yokoyama, "Bias-stress-induced increase in parasitic resistance of InP-based InAlAs/InGaAs HEMTs," Microelectronics Reliability, vol. 42, pp. 47-52, Jan. 2002.

[4] N. Hara, N. Okamoto, K. Imanishi, K. Sawada, T. Takahashi, K. Makiyama, and M. Takikawa, "Improvement in reliability of InP-based HEMTs by suppressing impact ionization," Proc. on 16th Int. Conf. on Indium Phosphide and Related Materials, Kagoshima, Japan, pp. 615-618, May 2004.

[5] T. Suemitsu, H. Yokoyama, T. Ishii, T. Enoki, G. Meneghesso, and E. Zanoni, "30-nm two-step recess gate InP-based InAlAs/InGaAs HEMTs," IEEE Trans. Electron Devices, vol. 49, no. 10, pp. 1694-1700, Oct. 2002. 
[6] T. Suemitsu, T. Ishii, and Y. Ishii, "Gate and recess engineering for ultrahigh-speed InP-based HEMTs," IEICE Trans. Electron., vol. E84C, no. 10, pp. 1283-1288, Oct. 2001.

[7] T. Suemitsu, T. Enoki, H. Yokoyama, and Y. Ishii, "Improved recessedgate structure for sub-0.1- $\mu$ m-gate InP-based high electron mobility transistors," Jpn. J. Appl. Phys., vol. 37, no. 3B, pp. 1365-1372, March 1998.

\section{Introduction}

A lot of studies have been carried out to find ways to improve the reliability of InP-based high electron mobility transistors (HEMTs) [1]. It has been shown that an InP etch stop layer, which was originally incorporated to control the etching depth of the gate recessing, has a surface passivation effect at the gate recess region, which has a great impact on device stability $[2,3]$. However, the drain resistance $\left(R_{d}\right)$ increases when both bias and thermal stress are applied to the sample. This failure mode has been studied for a long time and is still under investigation. Several groups have pointed out that hot carrier effects play a role in this failure mode [3, 4]. However, hot carrier effects alone cannot explain all phenomena related to the increase in $R_{d}$. Our previous study on InP HEMTs with different gate recess widths revealed that $R_{d}$ increases more in devices with a narrower gate recess after long-term bias-and-temperature (BT) stressing. This can be explained by the larger electric field present in the narrower gate recess devices leading to an enhanced hot carrier effects. However, the devices with a wide gate recess, when submitted to $\mathrm{BT}$ stress, also exhibited an overshoot in $R_{d}$, i.e., a rapid increase in $R_{d}$ is observed at the early stage of the BT stress, then $R_{d}$ reaches a maximum and then a continuous decrease in $R_{d}$ is observed for longer BT stress time [3]. Although widening the gate recess is a promising way to mitigate the hot-carrier-induced degradation, this overshoot in $R_{d}$ must be eliminated to make this approach practical.

In this letter, we report two-step-recess (TSR) gate InP HEMTs $[5,6,7]$ with a new process option suitable for producing a wide recess. In the resulting device structure, the gate recess reaches the ohmic edge and is completely covered with a passivation film. Though the gate recess is extremely wide, the cost to the device performance is negligible; the transconductance is $1 \mathrm{~S} / \mathrm{mm}$ and the cutoff frequency is $208 \mathrm{GHz}$ in $100-\mathrm{nm}$ gate devices. Moreover, the devices exhibit improved stability of $R_{d}$ compared to conventional TSR HEMTs.

\section{Device structure and fabrication}

The heterostructure grown by metal organic chemical vapor deposition is the same as that reported previously, i.e., n-InGaAs cap $\left(12 \mathrm{~nm}, 1 \times 10^{19} \mathrm{~cm}^{-3}\right)$, nInAlAs cap $\left(15 \mathrm{~nm}, 6 \times 10^{19} \mathrm{~cm}^{-3}\right)$, i-InP etch stopper $(6 \mathrm{~nm})$, i-InAlAs Schottky barrier $(10 \mathrm{~nm})$, Si planar-doped, i-InAlAs spacer $(3 \mathrm{~nm})$, i-InGaAs channel $(15 \mathrm{~nm})$, and i-InAlAs buffer $(200 \mathrm{~nm})$ layers on a (100) semi-insulating 
InP substrate [5]. The ternary alloys (InAlAs and InGaAs) are latticematched to InP. In this study, the fabrication process in [5] was followed, but the first recess etching, carried out with citric-acid based solution, was carried out before deposition of the $\mathrm{SiO}_{2} / \mathrm{SiN}_{x}$ passivation film. The resulting structure has two key features: One is a wide gate recess since the cap layers on the region between source and drain electrodes are almost completely etched off. The other is that in the gate recess the semiconductor surface (InP etch stopper) is completely covered with the $\mathrm{SiO}_{2} / \mathrm{SiN}_{x}$ passivation film as shown in Fig. 1 (called the fully passivated (FP) TSR hereafter). This is in contrast to the conventional gate recess, in which an air gap exists between the semiconductor surface and the passivation film. The gate-source and gate-drain distances are $500 \mathrm{~nm}$ and $1 \mu \mathrm{m}$, respectively. For comparison, devices made with the conventional TSR process, which has a recess length of $200 \mathrm{~nm}$ as estimated from the etching time [6], were prepared as controls. The gate length of the both types of devices is $100 \mathrm{~nm}$.
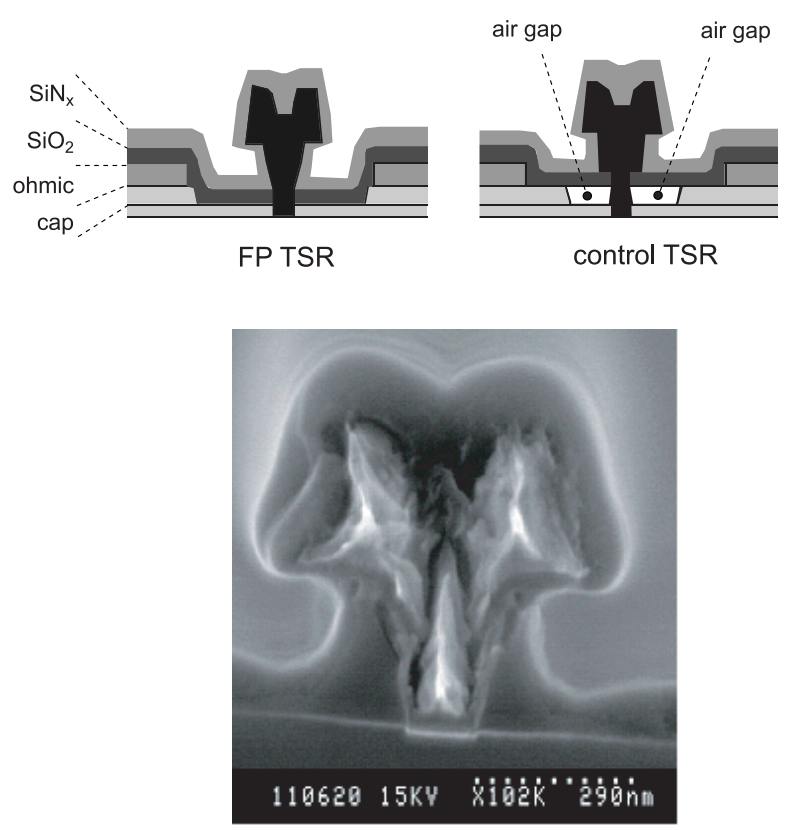

Fig. 1. Schematic cross section of FP TSR and control TSR gates [top] and scanning-electron-microscope image of a FP TSR gate [bottom].

\section{Results and discussion}

Typical output characteristics of the FP TSR devices are shown in Fig. 2. The extrinsic transconductance $\left(g_{m}\right)$ is $1 \mathrm{~S} / \mathrm{mm}$, which is smaller than that of the control TSR devices $(1.2 \mathrm{~S} / \mathrm{mm})$ because of the larger source resistance caused by the wider gate recess. However, the decrease in $g_{m}$ is minimized by the TSR gate because of the large carrier concentration in the gate recess [7]. In fact, the sheet resistance at the recess region is $273 \Omega /$ sq whereas it would be more than $400 \Omega /$ sq if it were a plain gate recess. The current gain cutoff 

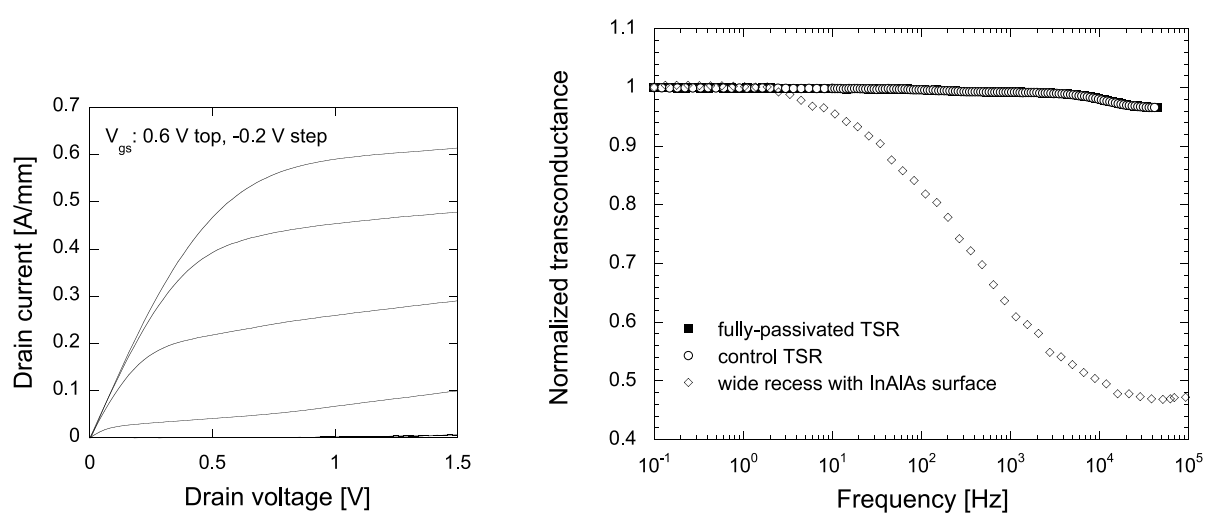

Fig. 2. Output characteristics of 100-nm InP HEMTs: [left] DC characteristics of FP TSR HEMTs. [right] The $g_{m}$ frequency dispersion of FP-TSR and control TSR HEMTs. Drain voltage is $0.1 \mathrm{~V}$. The result for a HEMT having a wide recess with InAlAs surface [5] is also shown.

frequencies of both types of devices are comparable; $208 \mathrm{GHz}$ and $215 \mathrm{GHz}$ for the FP and the control TSR devices, respectively. Thanks to the wider gate recess, the FP-TSR devices exhibit better off-state drain breakdown voltage $(5 \mathrm{~V})$ than control devices $(3.3 \mathrm{~V})$.

No kink effect is observed in the output characteristics of the new FP-TSR devices as demonstrated by Fig. 2 left. This indicates that the gate recess region is electrically stable despite it being as wide as about $500 \mathrm{~nm}$. The electrical stability of the recess region was also examined by the frequency dispersion of transconductance, $g_{m}(f)[2]$. The $g_{m}$ dispersion of the FP TSR devices is similar to that of the control TSR devices and the magnitude of the $g_{m}$ dispersion is negligibly small compared to that for plain gate devices having a wide recess with InAlAs surface [5]. These confirm that the passivation effect of the InP etch stop layer does work in the FP-TSR devices even though the recess region is wide and the InP etch stop layer experiences a plasma process when the passivation film is deposited.

For control TSR devices, the mechanical and chemical strength is a serious concern of the wide recess because of the air gap. Actually, a BT stress test has revealed unique features of the degradation mode of wide-recess devices: (1) the drain resistance $\left(R_{d}\right)$ increases more rapidly as the gate recess becomes wider; (2) for the narrow recess devices, $R_{d}$ increases monotonically but, for the wide recess ones, the increase in $R_{d}$ has an overshoot, i.e., $R_{d}$ sharply increases and then decreases; and (3) after long-term stress $(\sim 1000 \mathrm{~h})$, the devices with wider gate recess exhibit smaller drain resistance [3]. Such phenomena are thought to occur as a combined result of two degradation modes. The first degradation mode causes a monotonic increase in the drain resistance, which is more severe in devices with narrower recess, presumably due to hot carrier effects. The second is a recoverable degradation mode that causes the rapid increase and subsequent decrease in $R_{d}$. This degradation mode is visible only in wide-recess devices. Hence, if we can eliminate it, a 
wide recess could be a potential solution to the long-term reliability problem of InP HEMTs.

Figure 3 shows the changes in the drain resistance under BT stress (drain and gate voltages of 1.5 and $0 \mathrm{~V}$, respectively, in nitrogen ambient at $195^{\circ} \mathrm{C}$ ). The broken lines are for the control TSR devices and re-present the results in [3] - a sharp large increase in and overshoot of $R_{d}$ with a large dispersion in $R_{d}$ among devices throughout the BT stress test, particularly during the overshoot. On the other hand, the FP-TSR devices (solid lines) exhibit a much smaller increase in $R_{d}$ and no overshoot is observed. In addition to its magnitude, the uniformity of $R_{d}$ is maintained throughout the BT stress test. As mention above, our previous study reported that the overshoot in the $R_{d}$ increase becomes more apparent at wider gate recess. In this study, however, the FP-TSR devices had a wider gate recess than the control devices. Therefore, the wide recess itself does not the cause of the overshoot in $R_{d}$. The remarkable improvements obtained by means of the FP-TSR suggest a critical role of the air gap in the control devices, which does not exist in the FP-TSR devices.

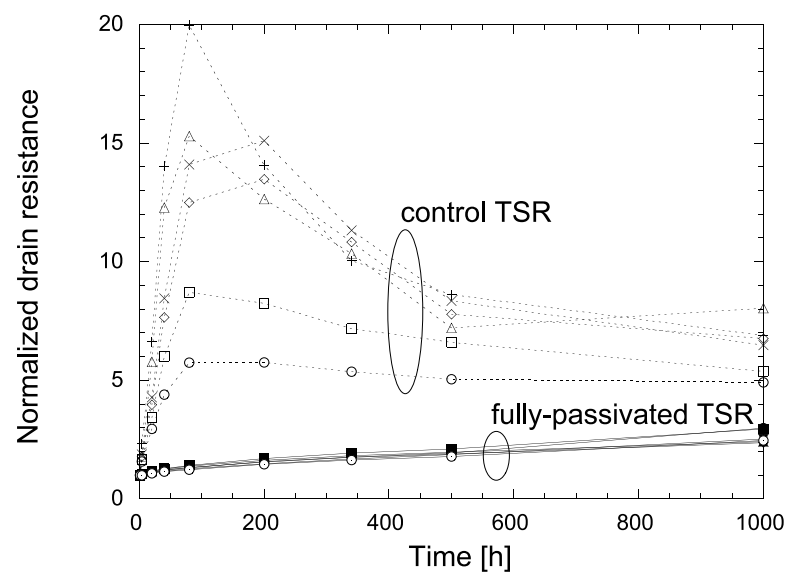

Fig. 3. Change in drain resistance of FP TSR and control TSR HEMTs. BT stress: Drain voltage of $1.5 \mathrm{~V}$ and ambient temperature of $195^{\circ} \mathrm{C}$.

\section{Conclusion}

An InP HEMT with a fully passivated (FP) two-step recess (TSR) exhibits improved stability of the drain resistance under bias-and-temperature stress. The new process can eliminate the air gap between the semiconductor surface and the passivation film in the gate recess region. This enables us to overcome a serious degradation mode appearing in the drain resistance of conventional HEMTs when a wide gate recess is produced. The transistor performance, $g_{m}=1 \mathrm{~S} / \mathrm{mm}$ and $f_{T}=208 \mathrm{GHz}$ for a gate length of $100 \mathrm{~nm}$, does not show a critical tradeoff compared to those of conventional HEMTs. 


\section{Acknowledgments}

The authors are grateful to M. Mitsuhashi for her assistance in the BT stress test. 